On Seriphos there is much copper slag and a number of galleries in the iron mine which may have been seeking veins of copper; such rejection of the iron ore might point to early working, though there is some evidence that mining was being practised here in the later centuries B.C.

On Paros, copper slag was found in an early bronze age tomb at Abyssos. Near Naussa is a small mine of malachite, much overgrown, with a gallery leading off at each end; all the ore had been removed, as is usual in prehistoric mines; but otherwise the evidence for its early date is weak.

On the early bronze age acropolis of Syra a piece of copper slag was found.

The cave of Chrysokamino has yielded early and middle bronze age pottery, and was probably a copper mine, though all the ore has been extracted ; slag, burnt clay and pieces of perforated crucible have been found outside. Gournia nearby was probably a manufacturing centre, considering how many moulds it contained, but Chrysokamino is too small to have supplied the whole of Crete with copper during the bronze age.

The copper mines of western Crete which are claimed as prehistoric were opened in Hellenistic times and continued until the Middle Ages. Much copper ore is also reported from Gavdos, but there is no reliable information as to when it was worked.

As to more distant sources, the direct evidence for bronze age mining in Cyprus is not strong, and it must be considered doubtful if the island supplied copper to the Aegean so early. The mines of Sinai are doubtful, the ore being apparently always poor, and it is likely that such copper as was produced was largely absorbed by the Bedouins, save at the times of Egyptian expeditions. The Kara Öyük tablets mention copper as an object of trade, but it is not common in western Anatolia. Italy seems to have been working copper sporadically from early times, and the Homeric Temesa is probably to be sought in this direction, whether Etruscan or south Italian ores were used. Largely, however, the Greeks seem to have used small local deposits which they completely exhausted.

\footnotetext{
Herodotus, 3, 57 ; cf. Bent, J. Hellenic Studies, 6, 195.

"Cf. Ardaillon, "Le Laurium"; also Davies, Man, 6, 1931.

stephanos, Cong. int. Archéologie, I, Athens, 216; 1905.

- For Siphnos see above.

- Freise, $Z$. Berg. Hittten, und Salinenwesen im preuss. Staat, 56 $347 ; 1908$.

- Paton and Myres, Geog. J., 9, $38 ; 1897$.

" "Iliad", ii, 856.

- Bosanquet, Rep. Brit. A88. Adv. Sci., 722, 1904. Athens Nat. Mus., Inventory No. 12445.
}

\title{
Electrical and Magnetic Units
}

$\mathrm{T}$ HE British Association has done a great service to the electrical industry by fostering the study of electrical standards and it was with considerable interest that Section $\mathrm{A}$ listened to two papers given by Sir Richard Glazebrook at the recent York meeting. Sir Richard has been an active member of the British Association committee on electrical standards which has laboured for a period of fifty years. In 1913 a single volume edited by Sir Frank Smith was published, giving the complete series of thirty-nine reports. Sir Richard was present at the York meeting in 1881 when Section A met at the same centreSt. Peter's School-and received the eighth interim report of the committee for constructing and issuing practical standards for use in electrical measurements. One of the papers* presented by him at the recent meeting showed the changes which have taken place in the British Association wire resistance coils over the period of half a century. The conclusion reached is that platinum is the most stable material to use for the construction of standard coils. This paper has already been referred to in NATURE (Oct. 22, 1932) and will be published in full in the Report of the British Association for 1932. The other paper $\dagger$ dealt with a topic which is still under discussion, namely, the definition of electric and magnetic units.

Sir Richard Glazebrook is president of the Com-

* Material Standards of Resistance : the British Association Coils, 1881-1932, By Sir Richsrd Glazebrook and Dr. L. Hartshorn.

Paris, July, 1932. By Sir Richard Glazebrook and Dr. Ezer Griffiths, No. 3296, Vol. 1301 mission of the International Union of Pure and Applied Physics which was set up by the General Assembly at Brussels in 1931 to consider the symbols, units and nomenclature used in physics.

The Commission at its first meeting decided that the most urgent problem awaiting solution was the definition of electrical and magnetic magnitudes. The first step taken therefore was to prepare a questionnaire which was circulated to the national committees working under the auspices of the Union of Physics in various countries.

The principal issues raised by this questionnaire related to :

(1) The basis on which a connected account of electromagnetic phenomena should rest. Should the starting point be Coulomb's law of force between magnetic poles or some other physical law?

(2) Should $\mu$ (the permeability) be treated as a quantity having dimensions in length, mass and time, or as a pure number? In other words, are $H$, the strength of a magnetic field, and $B$, the magnetic induction, quantities having different dimensions or are they quantities of the same kind?

The British reply to this questionnaire was agreed to at a meeting held in the rooms of the Royal Society in May last, at which were present, besides the members of the National Committee for Physics, representatives of the British Association Committee dealing with Magnetic Units and British representatives of the International Electrotechnical Commission, which had also been dealing with this matter. 
The British view is that:

(1) Coulomb's law is acceptable as a starting point.

(2) Permeability should be regarded as a quantity having dimensions.

Meanwhile, replies to the questionnaire had been received by the Commission from national committees abroad and from individuals; many of the latter submitted memoranda running into fifty typed pages. To advance matters a stage further it was decided to take advantage of the presence in Paris of a number of experts attending the Electrical Congress, to call an informal conference. This conference was attended by: Sir Richard Glazebrook (chairman), Prof. Wilberforce, Dr. Ezer Griffiths (secretary), all of Great Britain ; Profs. Abraham (secretary of the Union of Physics), Fabry, Paul Janet, Brylinski, Cotton, P. Bunet, Lienard (France); Prof. Verschaffelt (Belgium); Profs. van Staveren and van de Well (Holland); Prof. Wallot (Germany); Prof. Lombardi (Italy); Prof. Bjerknes (Norway); Profs. Kennelly and Pender, and Dr. Curtis (United States).

The conference had before it a summary of the replies received to the questionnaire and after consideration of these a series of proposals were submitted by the chairman. Some of these proposals were accepted unanimously and on others an informal vote was taken.

It was unanimously agreed that:

(1) Any system of units recommended must retain the eight internationally recognised practical units : joule, watt, coulomb, ampere, ohm, volt, farad, henry.

(2) The C.G.S. system is suitable for the physicist. (3) A system of practical units, including the above eight quantities, can be derived from these by multiplying the C.G.S. unit by appropriate powers of 10 .

One important proposition before the Conference dealt with the basis on which to build any system of magnetic units.

Should a start be made from:

(a) the force between two poles (coulomb).

(b) the force between two current-carrying elements (ampere) or

(c) the idea of 'flux'?

There was no decided majority in favour of any one of these. The view expressed by the British Committee was that the force between two elementary magnetic poles provides the most satisfactory basis. On the other hand, a number of French physicists were in favour of taking the force between two elements of current as the basis.

The chairman explained the system formulated by Maxwell, but with the proviso that $\mu_{0}$ and $K_{0}$ are both quantities having dimensions.

MAXWELL'S SYSTEM

$$
\begin{aligned}
& \text { Force }=\frac{\epsilon \epsilon^{\prime}}{\overline{K_{0} r^{2}}} \quad \cdots \quad . . \\
& \text { Force }=\frac{m m^{\prime}}{\mu_{0} r^{2}} \quad \ldots \quad . .
\end{aligned}
$$

$$
\begin{aligned}
& \text { Force }=\frac{m i \sin \theta d s}{A r^{2}} \\
& \text { Where } \frac{A^{2}}{\mu_{0} K_{0}}=(\text { Velocity })^{2}
\end{aligned}
$$

This velocity is shown by experiment to be the velocity of electromagnetic waves. Also $A$ is a constant for all media. Maxwell puts $A=1$ and alternatively :

$$
\begin{aligned}
& K_{0}=1 \text { Electrostatic system. } \\
& \mu_{0}=1 \text { Electromagnetic ", }
\end{aligned}
$$

In a vacuum we are to have

$$
F=m m^{\prime} / \mu_{0} r^{2}
$$

and in any other non-magnetic medium

$$
F=m m^{\prime} / \mu_{1} r^{2}
$$

where $\mu_{1}$ is characteristic of the medium.

For such media we have for the magnetic induction $B_{0}$ or $B_{1}$

$$
B_{0}=\mu_{0} H . \quad B_{1}=\mu_{1} H .
$$

where $H$ is the strength of the magnetic field.

Hence $B_{1}=\frac{\mu_{1}}{\mu_{0}} B_{0}=\mu B_{0}$

where $\mu$ is the specific permeability: a nondimensional quantity given by the ratio $\mu_{1} / \mu_{0}$.

In a magnetic medium we have $B_{1}=\mu_{1} H$ but in this case $\mu_{1}$ is no longer a constant; its value is given by $\mu_{0}\left(1+\frac{4 \pi I}{\mu_{0} H}\right)$, where $I$ is the intensity of magnetisation and $H$ is the field strength measured by the force on a unit pole placed at the centre of a long narrow tunnelshaped cylinder with its axis parallel to the lines of magnetisation.

The alternative $(b)$ is referred to as the electrodynamic system ; in this, following Ampere's equations, (2) and of (3) of Maxwell's system referred to above are replaced by

$$
\text { Force }=i^{\prime} d s^{\prime} \int \frac{[r i \sin \theta d s]}{r^{3}}
$$

or possibly

$$
\text { Force }=\mu_{0} i^{\prime} d s^{\prime} \int \frac{\left[r i \sin ^{2} \theta d s\right]}{r^{3}}
$$

Each equation represents the force on an element $i^{\prime} d s^{\prime}$ due to a closed circuit of which $d s$ is an element carrying a current $i$. The expression in the brackets represents a vector in a plane perpendicular to $r$ and $d s$ and these vectors are to bo compounded vectorially.

The other suggestion $(c)$ is that a system of units should be based on magnetic flux. The space inside a hollow anchor ring over the surface of which is wound a single layer of wire carrying a current, is found by experiments with iron filings or a magnetic needle to be in a peculiar condition, and the magnetic disturbance at each point within the ring has not only a direction but also a magnitude. The disturbance is said to be in the form of flux.

Both the electrodynamic system and the system based on flux spring from a desire to avoid the

No. 3296, Vor. 130] 
necessity of introducing the idea of an isolated magnetic pole.

Sir Richard Glazebrook in his paper to the British Association at York points out that an ammeter or a voltmeter depends for its effects on the forces between a current and a permanent magnet, whereas the object of both the systems $(b)$ and $(c)$ referred to above is to avoid reference to permanent magnetism. As he points out, we may measure a current by its electrolytic effects and having done so, its E.M.F. by the heat generated in a coil in a calorimeter. In practice, we have to connect the quantitiesso measured with the accepted units, the ampere and the volt, thus complicating our fundamental definitions with the value of the electro-chemical equivalent of silver, or, if preferred, hydrogen. He directs attention to those who object to Coulomb's law to Appendix C of the Second Report (Newcastle 1863) of the Electrical Standards Committee of the British Association. It is by Clerk Maxwell and Fleeming Jenkin and deals with the elementary relations between electrical measurements.

Those who favour the basis of the system of magnetic units as the force between the two elements of current were invited to put forward a consistent plan of a series of definitions of electrical and magnetic units.

Another topic which came up for discussion at Paris was-

"Are $B$ and $H$ quantities of the same kind and is their ratio $\mu$ a pure numeric ? Or should $\mu$ be treated as a dimensional quantity ?"

In submitting the point of view of the British
National Committee that $B$ and $H$ are quantities not of the same kind, Prof. Wilberforce pointed out that $B$ has been defined according to one method by Maxwell in the early part of his work when considering the electromagnetic system; later, when treating the possibilities of other systems, he defined $B$ in a different manner. According to Maxwell's first method, $B$ and $H$ would be quantities of the same dimensions and according to his second method they would be of different dimensions. Until it can be decided what method of definition is to be adopted, it is impossible to state whether $B$ and $H$ are to be looked upon as quantities of the same kind. The British view is based on the more general method following Maxwell's later work. The view of the Dutch Committee is that $B$ and $H$ are quantities of the same kind.

In the course of the discussion, the chairman referred to the fact that he was one of the last surviving pupils of Maxwell and he felt con. vinced from recollections of Maxwell's teaching that he was of the opinion that $B$ and $H$ were quantities of a different kind. When a vote was taken, nine were in favour of treating $B$ and $H$ as quantities of a different nature, whilst three were in favour of regarding $B$ and $H$ as quantities of the same nature.

Another issue raised was whether the factor $4 \pi / 10$ be retained in the definition of magnetomotive force. One speaker remarked that if one omits $4 \pi$ in one place it occurs elsewhere. The consensus of opinion was against its omission.

Ezer Grithiths.

\section{News and Views}

Sir Frank Dyson, K.B.E., F.R.S.

Sir Frank Dyson, Astronomer Royal, will terminate his official connexion with the Royal Observatory, Greenwich, on February 28. He went to Greenwich in 1894, when he was appointed a chief assistant. After retaining this position for ten years, he was appointed Astronomer Royal for Scotland. $\mathrm{He}$ returned to Greenwich after six years absence, being appointed as the successor of Sir William Christie. Throughout his career, Sir Frank has taken a keen interest in all the departments of the Observatory; it may perhaps be said that his greatest interest has lain in the determination of the proper motions of the fainter stars. In company with $\mathrm{Mr}$. W. G. Thackeray, he made a careful re-reduction of the catalogue of faint stars observed by Groombridge at Blackheath a century earlier; these were compared with recent Greenwich observations, providing proper motions of several thousands of faint stars. The later Greenwich catalogues have all been planned with the view of the determination of proper motions for successive zones of the sky. Sir Frank has been a keen observer of solar eclipses, obtaining successful results in 1900 (Portugal), 1901 (Sumatra), 1905 (Tunis), 1927 (England); he wrote a paper in the
Phil. Trans. for 1906, which is still regarded as providing the standard determination of coronal wave. lengths. It was also under his auspices that the expedition went to Brazil in 1919 to test the Einstein shift of starlight. Both the magnetic department and the time-service have been revolutionised in recent years; the former was moved to Abinger, as the electric railways in London were a disturbing factor. For the latter, a series of Shortt clocks in air-tight cases give very precise results; also daily comparisons with other observatories are made by wireless signals. Sir Frank has considered his successor in obtaining the provision of a new reversible transit-circle, which is nearing completion. The present circle is eighty years old, and its shutters are too narrow, not permitting free circulation of air.

\section{Dr. H. Spencer Jones, F.R.S.}

Dr. Harold Spencer Jones, His Majesty's Astronomer at the Cape, has been appointed Astronomer Royal in succession to Sir Frank Dyson, and will commence his duties next March. Dr. Spencer Jones is well-known at Greenwich, for he went there in 1913 and served for ten years as chief assistant. The study of optics is one of his favourite pursuits; No. 3296, Vol. 130] 